\title{
BIOPSY OF THE LEFT AURICLE IN MITRAL STENOSIS
}

\author{
BY \\ J. B. ENTICKNAP \\ From the Department of Pathology, Guy's Hospital
}

Received August 14, 1952

During the past three years operations upon the hearts of patients with mitral stenosis have been performed in increasing numbers (Baker et al., 1952). An opportunity has thus arisen to study by biopsy the changes in the heart and lungs at a time when, although the disease is fully established, the patient is still in a fairly good state of health. Previous morbid anatomical studies having been performed on post-mortem material, it has always been uncertain how much of the changes seen could be ascribed to the processes of death and, to a lesser extent, to autolysis. Furthermore, although many extensive investigations of cardiac histology have been made, very little attention has been paid to the tip of the left atrium. This paper deals with the changes found in this situation and a subsequent publication will describe those found in the lingular segment of the left lung. At least one other article has appeared purporting to show that histological evidence of rheumatic activity is commonly seen in auricular biopsies ((Kuschner et al., 1952) who report Aschoff bodies in 4 cases out of 11) and similar opinions have been expressed by other pathologists during public discussions. It is proposed to examine the validity of these statements in the light of the findings in a large series of patients.

\section{MATERIAL AND MethodS}

The main observations have been made on 71 specimens of cardiac muscle removed by Mr. R. C. Brock during the course of operations for mitral valvotomy. A An incision is made into the tip of the left auricle (auricular appendage) at the beginning of this operation and through it the gloved finger is inserted. The duration of the manipulations varies from 10 to 30 minutes, during which time ether is the main anæsthetic agent, and at their conclusion the piece of tissue surrounding the wound is excised and fixed in neutral formol saline. No specimens were received from 16 of the cases of the Guy's Hospital operation series but this is not thought to have caused significant selection. All those received so far have been included in this survey.

Control material has been obtained from three sources. Human myocardium attached to fragments of fibrous tissue from 3 cases of congenital pulmonary stenosis which had been removed under similar conditions is the only material that is strictly comparable. Human auricles were obtained post mortem from a number of cases of rheumatic heart disease including four fatalities during the first week after operation and one after two months: also from 12 subjects with no evidence or history of heart disease and from 3 with benign essential hypertension, who had died from causes other than heart failure. Experimental animals were the third source, anatomically comparable specimens being obtained from 7 dogs, 5 rabbits, and 12 guinea-pigs. The dogs and half of the guinea-pigs were killed while under a similar anæsthetic to that used during the surgical operation and the rabbits and the remaining guinea-pigs by fracture of their cervical spines.

Histological methods. The tissue was embedded in paraffin wax so that, in the majority of instances, the resulting section showed the complete circumference of the auricle. In a few of them it was so arranged that the cut ends of the surgical incision were available for inspection. One section from each case was stained with Mayer's hemalum and eosin and where necessary Lendrum's modification of Mallory's trichrome method (Lendrum, 1949), Weigert's elastic tissue method, Perls's reaction for iron, Mallory's phosphotungstic acid hematoxylin, Van Gieson's stain and Gordon and Sweet's method for reticulin (1936) 
were used. Serial sections extending through about $1 \mathrm{~mm}$. of tissue were cut in all cases in which the appearances in the first section examined in any way resembled those of acute rheumatism and in those in which organizing thrombi were present. A few frozen sections stained with oil red revealed no excess of fat.

\section{RESUlts}

These are set out in Tables I and II from which it can be seen that, in addition to " probable" and " possible" rheumatic lesions, a number of non-specific changes was present. Before describing these abnormalities it is necessary to define some of the terms used.

Cellular foci were said to be present when one or more localized collections of not less than five chronic inflammatory cells were seen in the interstitial tissue in any section. In Table I foci containing Aschoff cells have been recorded separately (see below).

Cubing of the endothelium is a term used to describe swelling of a sheet of surface cells so that they come to resemble columnar epithelium rather than the flat cells normally found on serous membranes.

Acute myocarditis was diagnosed when an inflammatory exudate consisting largely of polymorphonuclear neutrophil cells was present in the interstices of the myocardium.

EEdema of the myocardium implies separation of the muscle cells by clear spaces that presumably contained fluid in vivo and is distinct from the intra-cellullar vacuolation implied by the term next defined.

Cytoplasmic irregularity is a term used for a condition practically confined to and almost uniformly present in biopsy specimens. In sections stained with eosin the cytoplasm of the muscle cells shows all variations from a foamy vacuolated appearance that is almost unstained to an intensely coloured even cherry red or deep pink such as is commonly seen in post-mortem sections of ventricular myocardium. The appearances are even more striking if Lendrum's stain is used, when the different components of this composite stain may appear in the cytoplasm in almost any combination.

Abnormal nuclear forms. Giant nuclei are darkly stained and may be swollen up to as much as $50 \mu$ in diameter. The bizarre forms are even darker and have an irregular crinkled outline with a plicated appearance that suggests that they have been formed from collapse and shrinkage of the giant forms.

Rheumatic lesions have been diagnosed, for the purposes of this discussion, whenever Aschoff cells were found. The comprehensive review by Gross and Ehrlich (1934) of the appearances shown by Aschoff bodies in human hearts provides the most suitable standard of reference and the definitions there given have been accepted. They state that the Aschoff cell is large and has a variable amount of amphophilic or basophilic cytoplasm with ragged edges. Commonly the single oval nucleus shows irregular aggregations of chromatin and a dense nuclear membrane that is often folded upon itself, this being the fibrocytoid type of nucleus. In others one of the chromatin masses is stellate and larger than the rest, giving the appearance of the owl-eyed type. Either form of nucleus may become pycnotic. When these cells were arranged in groups close to which there was some fragmentation of collagen giving an appearance fairly closely resembling an Aschoff body, usually of the mosaic type, a "probable" rheumatic lesion was diagnosed. In those cases in which the cells were diffusely scattered throughout the interstitial tissue in no particular relation to one another or to collagenous changes the diagnosis of a "possible" rheumatic lesion was made.

Intimal thickenings were described as cellular when they showed the presence of lymphocytes, newly formed capillaries or large numbers of fibrocyte nuclei and as acellular when the increase of intimal bulk was due solely to homogeneous hyaline material.

\section{Changes in the Pericardium}

Irregularity of the surface due to amorphous particles, fibrin flakes, and red cells was common and a few hæmorrhages were seen; these probably resulted from operative trauma. Most of the other findings were seen with about the same frequency in the biopsy and necropsy sections but the cubing of the endothelium and the appearance of arteriolitis in a coronary vessel require further comment. The former change was much commoner in the post-mortem sections from non-rheumatic subjects and does not therefore appear to be related to this form of heart disease. The latter occurred in a patient who had no clinical indications of active disease and whose subsequent course has been in no way eventful. The lingular biopsy was substantially normal and the only other finding in the auricle was some round-celled infiltration around the vessels and elsewhere in the pericardial fat. It must therefore remain an unexplained curiosity. No Aschoff cells were seen in the pericardium in any of the sections. 
Changes in the Myocardium (Table I)

It may at once be emphasized that no " probable " rheumatic lesions were found in this situation in any of the specimens, although a few separate Aschoff cells were seen in one case and small lymphocytic foci in five others. Acute myocarditis was seen once and its significance is as obscure as that of the arteriolitis found in the pericardium. The relative absence of fibrosis is also rather remarkable. Generalized cytoplasmic and nuclear changes were present in most of the biopsy specimens. In two cases nuclear changes were seen in the presence of homogeneous cytoplasm and in another the nuclei were entirely regular in appearance while the cytoplasm showed great irregularity. Identical cytoplasmic and nuclear appearances were seen in one of the three fragments of

TABLE I

Changes in the Left Atrial Biopsies from Patients with Mitral Stenosis

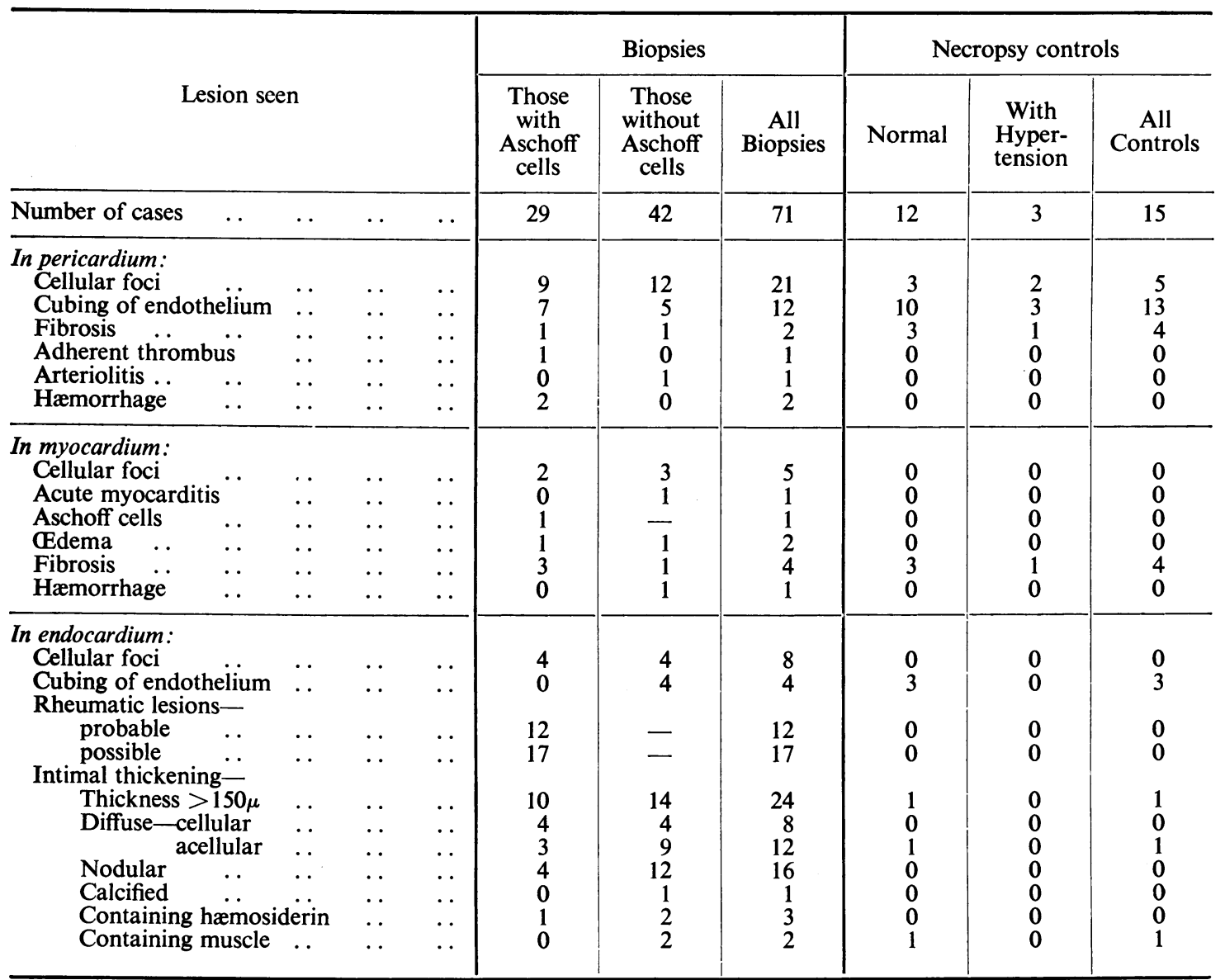

myocardium from patients with congenital heart disease. Staining with oil red showed that the cytoplasmic changes were not due to the presence of intra-cellular fat. Sections passing through the incised edge of the auricular wound showed that in this region the myocardial cytoplasm invariably had the homogeneous appearance customarily seen in post-mortem material. The necropsy specimens examined as controls to this series, with one exception, showed it, and there was no difference between those with rheumatic carditis, including one examined within four hours of death, and those without. Although the appearances of animal specimens are never identical with those of human tissues, comparable appearances were seen in one dog killed with ether from which the 
auricles were removed twenty minutes post mortem and in a single guinea-pig killed by this method, as well as in one that was bled to death. Similar results were found for the occurrence of abnormal nuclear forms.

\section{Changes in the ENDOCARdium aNd Lumen}

The most important changes were seen in these sites and they fall into three groups. The first two, intimal thickening and the presence of organizing thrombi, are in all probability interrelated, and the third group-lesions, the appearance of which suggests the presence of active acute rheumatism -is that to which most interest is attached.

Intimal thickening was seen in both nodular and diffuse forms. In order more precisely to evaluate the generalized form of thickening measurements of intimal thickness were made on all specimens examined and a normal range established. Four measurements were made at right angles to each other of the distance from the lumen to the border of the underlying muscle, except in those few cases in which the embedding process had caused an artificial separation, when the thickness of the separated intima was measured. In addition to this precaution, measurement of nodular thickenings and of portions of intima containing inflammatory exudate was avoided. The normal mean thickness so established was $80 \mu$ (Table II) with a standard deviation of $\pm 35 \mu$,

TABLE II

The Relationship between the Presence of Aschoff Cells in the Endocardium and of Organizing Thrombi IN THE AURICle

\begin{tabular}{cc|c|c|c}
\hline & & $\begin{array}{c}\text { Auricles } \\
\text { containing } \\
\text { thrombi }\end{array}$ & $\begin{array}{c}\text { Auricles } \\
\text { not } \\
\text { containing } \\
\text { thrombi }\end{array}$ & Total \\
\hline Probable Rheumatic lesions present &. & 4 & 8 & 12 \\
\hline Possible Rheumatic lesions present .. &.. & 6 & 11 & 17 \\
\hline No evidence of Rheumatic activity .. &.. & 21 & 21 & 42 \\
\hline
\end{tabular}

giving an upper limit of normal $(p=0.05)$ of $150 \mu$. In about one-third of the biopsy specimens $(32 \%)$ the endocardium was thicker than this, but the proportion of thrombi or Aschoff cells was no greater in the sections from these cases than in those from the other two-thirds, nor was a history of auricular fibrillation any commoner in them. In three cases a positive Perl's reaction proclaimed the presence of blood at some stage in the development of the thickenings and in another some focal calcification had taken place. In general the nodular thickenings were entirely composed of elastic tissue while the diffuse thickenings showed variable proportions of loosely arranged and compact parallel collagen bundles with some elastic fibres. In two cases the presence of muscle cells in the thickened endocardium was rather surprising.

An interesting incidental discovery was that the endocardium of the left auricle was approximately four times as thick as that of the right (mean $22 \mu$, range 5-59 $\mu$ ). The thickened endocardia of mitral stenosis subjects examined post mortem showed the same ratio.

Organizing thrombi were present in nearly half the cases $(43 \%)$ but there is no significant difference in the mean endocardial thickness of the cases with and those without them (Table II). It is not, however, possible to say that these cases are the only ones that have ever had thrombi within their auricles and, indeed, it is rather unlikely that this is so. All stages of organization were seen so that in several cases it was difficult to decide whether to classify the appearances as organizing 
thrombi or as endothelial thickenings. The presence of hæmosiderin has usually decided the issue in favour of a thrombus but in two cases this pigment was present in a typical acellular thickening. Further, it has been possible in several cases to show by serial section that typical cellular thickenings were directly continuous with an area of organizing thrombus (Fig. 1). In others endothelialization of plaque-like clot has been seen taking place over quite a large area.

Lesions suggestive of active acute rheumatism. In 4 cases cubing of the endothelium was seen, but here, as in the pericardium, this was commoner in the necropsy specimens and so is probably of no specific significance. Cellular foci consisting mainly of lymphocytes, although in a few cases plasma cells were seen, were present in 8 biopsy cases but not in any of the controls. Both "probable" and "possible" rheumatic lesions occurred with about the same frequency (12 and 17 cases respectively) (Fig. 2 and 3), and were rather commoner in those auricles that did not contain organizing thrombi (Table II). Fragmentation of collagen was present in some of these lesions but it resulted from simple loss of continuity of fibrils and was unassociated with the deep red amorphous appearance characteristic of fibrinoid

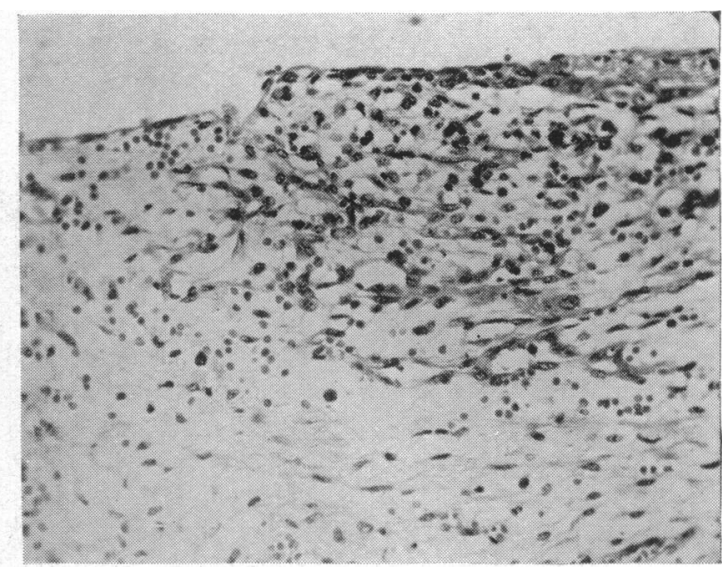

Fig. 1.-Cellular intimal thickening in continuity with organizing thrombus over which endothelium is growing. All preparations were stained with hematoxylin and eosin and were photographed at a standard magnification of $\times 205$. The prints have been mounted parallel to the line of the endothelium. when stained by eosin. Lendrum's stain did occasionally show replacement of the blue colour of healthy collagen by the yellow of the picric acid component but silver impregnation failed to reveal any argyrophil fibrils. It may then be emphasized that in no case was typical fibrinoid change of collagen seen.

Association of histological findings with evidence of sub-clinical acute rheumatism. As none of these patients had any clinical evidence of acute rheumatism at the time of operation an attempt has been made to estimate sub-clinical activity by an assessment of pre-operative temperature, erythrocyte sedimentation rate, and electrocardiogram. An unexplained temperature of over $99^{\circ} \mathrm{F}$., an E.S.R. (Westergren) of above $5 \mathrm{~mm}$. per hour in men and $10 \mathrm{~mm}$. per hour in women, or an electrocardiographic abnormality of which the commonest was temporary post-operative fibrillation, were each given one mark. The average score for each of three groups of patients was calculated: those with nodular "probable" lesions, those with "possible " lesions and those in whom no Aschoff cells were found. The results were 1 mark for the first group, 0.5 marks for the second group, and 0.8 marks, intermediate between the other two, for the third group.

\section{Discussion}

This investigation of the abnormalities seen in auricles removed from patients with mitral stenosis in whom there was no clinical evidence of either rheumatic carditis or cardiac failure has a two-fold purpose. The first is to throw some light on the thickening of the intima that is so commonly seen in healed rheumatic carditis, both in biopsy specimens and at autopsy, and the second is to try to evaluate the histological appearances that, despite the clinical findings, closely resemble the Aschoff bodies of acute rheumatism.

Intimal thickening. Of the two purposes the former has, at least in part, been achieved. Some of the appearances seen (Fig. 1) leave no doubt that incorporation of tissue derived from the organization of a thrombus may result in thickening of the intima and the presence of hæmosiderin in otherwise typical acellular thickenings suggests that many more of them may develop in a 

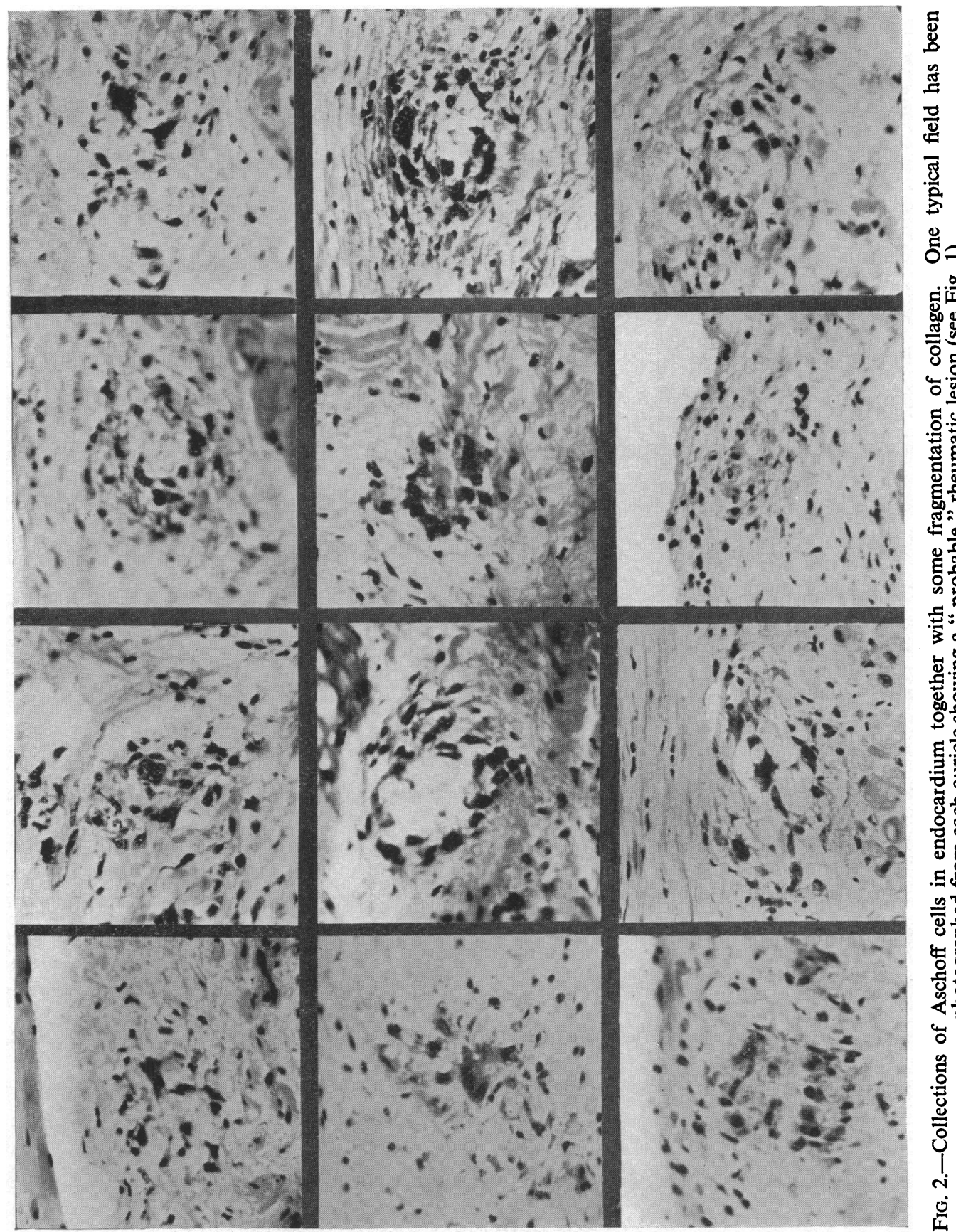
similar fashion. The frequent occurrence of elastic tissue in these lesions also suggests the same pathogenesis, since it regularly develops in organizing autogeneous thrombi in, for example, the pulmonary circulation of the rabbit (Heard, 1952). It is, however, improbable that organization of thrombi explains all these examples of intimal thickening, for the inflammatory exudates occurred predominantly in the subintimal tissue and would almost certainly give rise to fibrosis in this situation

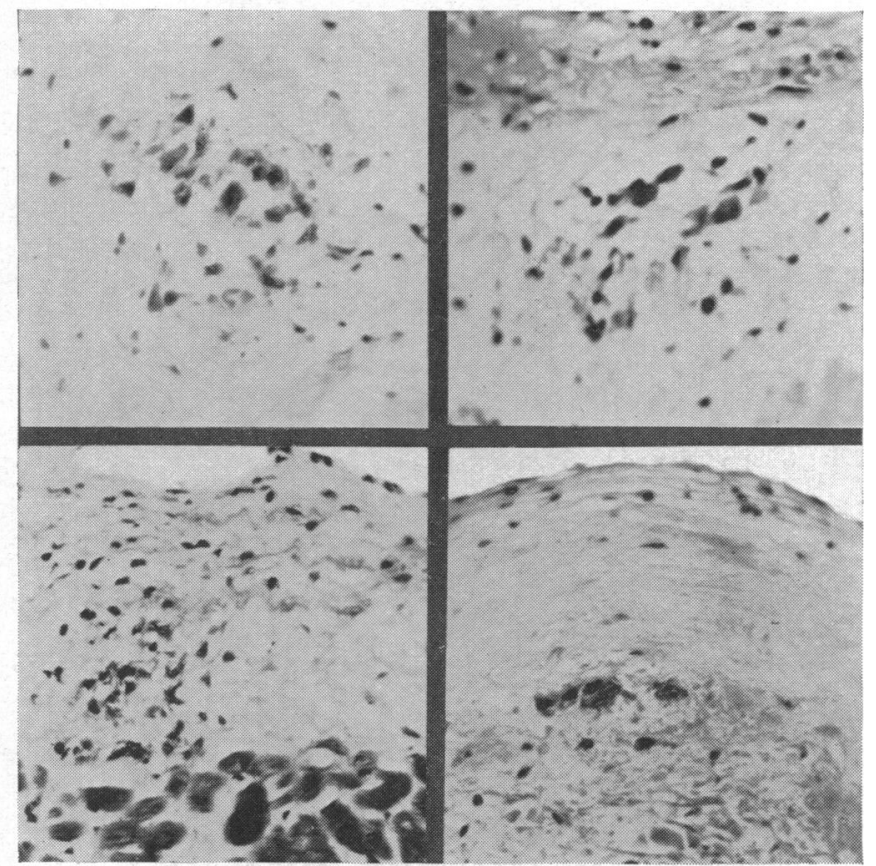

FIG. 3.-Aschoff cells in the endocardium not associated with collagenous changes. One photograph from each of 4 representative auricles showing " possible" rheumatic lesions (see Fig. 1).

on healing. It is also unlikely that the muscle cells seen in some of these thickenings were derived from metamorphosis of blood clot, the probable explanation of their presence there being that they were sequestrated from the main bulk of the myocardium as a result of inflammatory fibrosis. Finally, the mean intimal thickness in the auricles containing clot was no more than in those that did not. It may therefore be concluded that many of the intimal thickenings seen, the nodular ones in particular, are in fact derived from organization of thrombi, although this is probably not the explanation of all of them.

\section{Appearances Suggéstive of Inflammatory Disease}

The cytoplasmic and nuclear changes in the cardiac muscle cells were, at first sight, some of the most conspicuous abnormalities in these sections and the clear-cut separation, between the biopsy specimens that showed them and the normal necropsy specimens that did not, suggested that they were of diagnostic significance. From the findings reported it may, however, be concluded that these appearances were largely, if not entirely, due to the fact that specimens were fixed before autolysis had produced much change in the cytoplasm and that they are not therefore significant of inflammatory or other disease.

Cubing of the endothelial cells is clearly unrelated to rheumatic carditis in these specimens, 
because it was even commoner in the necropsy controls than in the biopsies and in the latter it was more frequently seen in the absence than in the presence of Aschoff cells.

Cellular foci not containing Aschoff cells were seen with equal frequency in cases with " rheumatic" lesions and those without. They were not uncommon in the pericardium in the control specimens so, although their significance is uncertain, they, too, are probably unrelated to rheumatic carditis.

Acute inflammatory lesions were seen in two cases, neither of which contained any Aschoff cells, but as there are no relevant clinical findings their significance is too uncertain for conclusions to be drawn from this observation.

Since it appears that none of the above assist in evaluating the infiltrations containing Aschoff cells seen in about a third of the biopsies, their intrinsic nature and situation and their association with organizing thrombi and with abnormal results to certain clinical tests will be considered in the hope that they may do so.

The minute structure of these lesions is not that of typical Aschoff bodies. All the " probable" and "possible" rheumatic lesions have been so diagnosed because they showed "mesenchymal cells" (Gross and Ehrlich, 1934) with irregular outline of which these authors state that "The most frequently occurring . . . structure of the Aschoff body is the basophilic cytoplasm with the ragged edges." They also state, however, that " no single component . . . (is) . . . by itself . . . a sufficiently characteristic feature to identify the Aschoff body " so that it deserves emphasis that this was, in fact, the only common feature of the lesions seen in these sections. Furthermore, swelling and fragmentation of collagen was seen in less than half of the "rheumatic lesions " after employing a variety of techniques specifically for the demonstration of this phenomenon. Those in which it was demonstrated are those termed "probable" lesions but it is noteworthy that in none of them was any substance with the typical tinctorial properties of fibrinoid seen. Opinions on the importance of this material in the histology of rheumatic disease are many and varied and seem to have been conditioned, at least in part, by whether the author was mainly concerned with human or experimental lesions. Thus Klinge (1933) places great importance on this substance which he illustrates so beautifully: while Gross and Ehrlich, on the other hand, in their very competent review of the lesions seen in human hearts say that " contrary to what has been stated in the literature [no references are given at this point, J.B.E.] these lesions rarely, if ever, show a fibrin constituent." Although not explicitly stated, the text suggests that this statement refers to a substance with the tinctorial properties of fibrin and is in fact an allusion to the absence of fibrinoid from the lesions that they examined. While the findings from this series agree very closely with this last statement the consensus of modern opinion is undoubtedly that true rheumatic lesions usually do show fibrinoid change of collagen (Glynn and Loewi, 1952) and the absence of it from a large series of lesions does strongly suggest that they are not indicative of active disease.

In summary these arbitrarily defined "probable" and "possible" rheumatic lesions are not histologically identical with, but do somewhat resemble, true Aschoff bodies. The former, which occurred in about one-sixth of the cases, did so rather more closely than the latter.

The distribution of these lesions, which were with one exception confined to the endocardium, is also somewhat unusual. Gross and Ehrlich (1935) examined necropsy specimens of the base of the posterior and medial walls of the left atria from 87 cases of rheumatic carditis in all stages of activity and found Aschoff bodies occurring with approximately equal frequency in both endoand myocardium. In their earlier study (1934) of the whole heart in active rheumatism they were able in all cases to find bodies in the myocardium and, indeed, confined their attention to these, believing that they were less likely to be affected by adventitious biological and histological factors. They further state that " legitimate doubts exist concerning the identity of these lesions (in the endocardium) with those found in the interstitial tissue of the myocardium"- -doubts which the findings in this series do nothing to dispel. This most important difference in the distribution of the lesions in the two series strongly suggests that they are not identical and that those found in the endocardium of the biopsies from the tip of the appendage of the atrium may have little of the 
significance of the Aschoff bodies found elsewhere in the heart in the post-mortem studies of earlier authors.

Organizing thrombi occurred about twice as frequently in the biopsies in which Aschoff cells were not seen as in those in which they were and Kuschner et al. (1952) reported that thrombi were absent from their four cases with Aschoff bodies. It is improbable, therefore, that the presence of these cells is a reaction to the presence of thrombus in the lumen, as sometimes appears from their close relationship in the sections, but it is difficult to draw any particular conclusion from this observation.

Association of these lesions with the occurrence of "sub-clinical" rheumatic phenomena did not occur with a frequency that is significant but the results are sufficiently suggestive to justify an investigation using more complex clinico-pathological methods designed to make a more precise evaluation of the rheumatic state. The salient fact is, however-and it cannot be right to overlook it in favour of any histological signs, however striking, or of the results of clinical tests, however conclusive they may appear to be in the light of current opinion - that not only did these cases have no overt clinical signs of acute rheumatism but that they were selected for operation out of many cases examined with this end in view during a period in which it was held that active carditis was a contra-indication to that form of treatment. It can therefore be said with certainty, that these patients were not suffering from acute rheumatism, as at present understood by leading clinicians, a fact that cannot be over-emphasized in deciding the significance of these lesions.

In conclusion these lesions that were found in patients who were not suffering from clinical rheumatic fever did not have the typical structure of Aschoff bodies, and were not distributed as Aschoff bodies usually are throughout all three layers of the heart. If, however, the histological signs be accepted as representing Aschoff bodies either the specific nature of these lesions must be doubted or the clinical state to which they are supposed to correspond must be redefined. In view of $(a)$ the atypical structure of these lesions, (b) the subsequent course of these patients which has not in any way resembled that of rheumatic fever, $(c)$ the fact that the frequency with which these lesions were found in acknowledged acute cases has varied from 32 per cent (Libman, 1923) to 97 per cent (Thayer, 1931), and $(d)$ the fact that there is still some doubt whether these lesions are indeed specific, it must surely be concluded that the findings in the few biopsies of heart muscle that have so far been examined, while certainly justifying doubts of the specific nature of Aschoff bodies, do not, on the other hand, justify redefining acute rheumatic fever. It is suggested that the best course is to regard the appearances seen as a new finding, the significance of which can only be elucidated by prolonged clinico-pathological investigation of, in particular, the subsequent progress of the disease and morbid-anatomical changes in the hearts in which they were found.

\section{SUMMARY}

Biopsy specimens of muscle from the left atrium of 71 patients with mitral stenosis have been examined and the results are reported.

Thickening of the intima was frequently found and was, in some cases at least, due to incorporation of organizing thrombi.

Lesions resembling Aschoff bodies were seen in the endocardium in about a third of the cases but their significance is uncertain. Reasons are given for believing that the appearances seen do not indicate active acute rheumatism. It is suggested that prolonged clinico-pathological investigation is necessary before they can be interpreted with certainty.

I wish to thank Mr. P. Brunnen for providing me with histological material from the hearts of dogs.

\section{REFERENCES}

Baker, C. G., Brock, R. C., Campbell, M., and Wood, P. (1952). Brit. med. J., 1, 1043.

Glynn, L. E., and Loewi, G. (1952). J. Path. Bact., 64, 329.

Gordon, H., and Sweets, B. S. (1936). Amer. J. Path., 12, 545. 
Gross, L., and Ehrlich, J. C. (1934). Amer. J. Path., 10, 467.

- (1935). Amer. J. Path., 11, 711.

Heard, B. E. (1952). J. Path. Bact., 64, 13.

Klinge, F. (1933). Ergebn. allgemn. Path., 27, 1.

Kuschner, M., Ferrer, M. I., Harvey, R.M., and Wylie, R. H. (1952). Amer. Heart J., 43, 286.

Lendrum, A. C. (1949). J. Path. Bact., 61, 443.

Libman, E. (1923). J. Amer. med. Assoc., 80, 813.

Thayer, W. S., (1931). Edinburgh med. J., 38, 237. 